\title{
CpG- and LPS-activated MAPK signaling in in vitro cultured salmon (Salmo salar) mononuclear phagocytes
}

\author{
Dimitar B. Iliev ${ }^{\mathrm{a}}$,Tom Hansen ${ }^{\mathrm{a}}$, Sven Martin Jørgensen ${ }^{\mathrm{b}}$, Aleksei Krasnov ${ }^{\mathrm{b}}$
}

\& Jorunn B. Jørgensen ${ }^{\mathrm{a}^{*}}$

\author{
${ }^{\text {a }}$ The Norwegian College of Fishery Science, University of Troms $\varnothing$, N-9037 Troms $\varnothing$, Norway \\ ${ }^{\text {b }}$ Nofima Marin AS, N-1432 Ås, Norway, \\ * Corresponding author: prof. Jorunn B. Jørgensen \\ Tel.: +4777646716 \\ Fax: +4777646020 \\ E-mail address: jorunn.jorgensen@ uit.no
}

Abbreviations: MAPK, mitogen-activated protein kinase; PAMPs, pathogen-associated molecular patterns; TLRs, the toll-like receptors; LPS, lipopolysaccharide; JNK, c-Jun NH terminal kinase; ERK, extracellular signal-regulated kinase; ODNs, oligodeoxynucleotides, IL-1 $\beta$, interleukin-1 $\beta$; MKK, MAPK kinase; MK2, MAPK-activated protein kinase2; DCs, dendritic cells; NFkB, nuclear factor kappa B; NRF2, nuclear factor (erythroid-derived 2)-like 2; PI3K, phosphatidylinositol 3-kinase; WB, Western blot; M-CSF, macrophage colony stimulating factor; HRP, horseradish peroxidase; eEF2, eukaryotic elongation factor 2; FBS, foetal bovine serum;

Keywords: mitogen-activated protein kinase (MAPK); CpG oligodeoxynucleotides (ODNs); lipopolysaccharide (LPS); Atlantic salmon (Salmo salar); mononuclear phagocytes 


\section{Abstract}

The Mitogen-Activated Protein Kinases (MAPK) are involved in transmitting intracellular signals downstream of diverse cell surface receptors and mediate the response to ligands such as growth factors, hormones and cytokines. In addition, MAPK are critically involved in the innate immune response to pathogen-derived substances, commonly referred to as pathogenassociated molecular patterns (PAMPs), such as bacterial lipopolysaccharide (LPS) and bacterial DNA rich in CpG dinucleotides. Currently, a great deal of knowledge is available about the involvement of MAPK in the innate immune response to PAMPs in mammals; however, little is known about the role of the different MAPK classes in the immune response to PAMPs in lower vertebrates. In the current study, p38 phosphorylation was induced by CpG oligonucleotides (ODNs) and LPS in primary salmon mononuclear phagocytes. Pretreatment of the cells with a p38 inhibitor (SB203580) blocked the PAMP-induced p38 activity and suppressed the upregulation of most of the CpG- and LPS-induced transcripts highlighting the role of this kinase in the salmon innate immune response to PAMPs. In contrast to $\mathrm{p} 38$, the phosphorylation of extracellular signal-regulated kinase (ERK), a MAPK involved primarily in response to mitogens, was high in resting cells and, surprisingly, incubation with both $\mathrm{CpG}$ and control ODNs downregulated the phospho-ERK levels independently of p38 activation. The basal phospho-ERK level and the CpG-inducible p38 phosphorylation were greatly influenced by the length of in vitro incubation. The basal phospho-ERK level increased gradually throughout a 5-day culture period and was PI3Kdependent as demonstrated by its sensitivity to wortmannin suggesting it is influenced by growth factors. Overall these data indicate that both basal and PAMP-induced activity of MAPKs might be greatly influenced by the differentiation status of salmon mononuclear phagocytes. 


\section{Introduction}

Ligands for innate immune receptors such as the toll-like receptors (TLRs) activate leukocytes by triggering complex intracellular signaling cascades. In mammals, it has been shown that TLR ligands, such as LPS and DNA rich in unmethylated CpG dinucleotides activate all major groups of MAPK in leukocytes, including monocytes, macrophages and dendritic cells (DCs) ([1], [2, 3] [4]). The major groups of MAPK include the extracellular signal regulated kinase (ERK), the c-Jun NH terminal kinase (JNK), and the p38 MAP kinase. Upon activation, MAPK are phosphorylated on the threonine (Thr) and tyrosine (Tyr) residues at their Thr-Xaa-Tyr (TXY) motif found in a highly conserved activation loop near the active site. The ERK signaling is activated mainly by mitogenic stimuli such as growth factors [3] .On the other hand, JNK and p38 pathways are activated by stress stimuli such as UV irradiation and osmotic shock, and by proinflammatory cytokines [5] The MAPK proteins and functions are well conserved across vertebrates and it has been demonstrated that in lower vertebrates, TLR ligands such as LPS and CpG ODNs, activate the innate immune response through MAPK. More specifically, LPS, CpG ODNs and recombinant trout interleukin-1 $\beta$ (IL-1 $\beta$ )-induced phosphorylation of endogenous p38 in salmon head kidney macrophages in a dose-dependent manner[6]. In addition, in whole salmon head kidney leukocyte cultures, p38 activation was shown to be crucial for LPS induced upregulation of immune genes including IL1- $\beta$, TNF, COX2 and, to a lesser extent, CD83 [7]

A major goal of the current study has been to further investigate the involvement of p38 in the response of salmon mononuclear phagocytes to LPS and CpG ODNs. Cultures of primary cells were stimulated with E. coli LPS and CpG ODNs alone or in the presence of a chemical inhibitor of p38 (SB203580). This inhibitor has been widely used in mammalian 
systems [8]; [9], however, data about its efficacy and specificity in lower vertebrates is scarce. In the current study, SB203580 was able to completely abolish the CpG- and LPS-induced p38 activity as determined by analysis of MAPK-activated protein kinase 2 (MK2, a direct target of p38) phosphorylation. A microarray analysis demonstrated that the upregulation of most of the CpG and LPS-induced genes was p38-dependent, highlighting the importance of the p38 kinase for the innate immune response of salmon leukocytes against pathogens.

Additional data indicate that the MAPK activation by PAMPs is affected by a PI3Kdependent mechanism and the differentiation of the cells. 


\section{Materials and methods}

\subsection{Fish and reagents}

Non-vaccinated healthy Atlantic salmon, Salmo salar L., strain Aquagen standard (Aquagen, Kyrksæterøra, Norway), 500-1000 g, was obtained from Troms $\varnothing$ Aquaculture Research Station (Troms $\varnothing$, Norway). The fish were kept at $10^{\circ} \mathrm{C}$ in tanks supplied with running filtered sea water and were fed on commercial dry food. All experiments were performed according to the guidelines from the national committee for animal experimentation (Forsøksdyrutvalget, Norway).

The phosphorothioate-modified ODNs were purchased from Thermo Scientific. The sequences are as follows, where phosphorothioate modifications are marked with * : CpG-B:

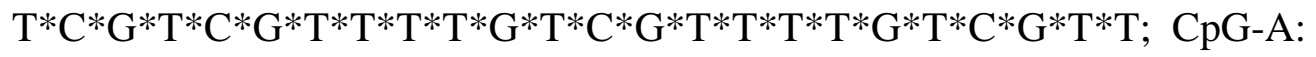

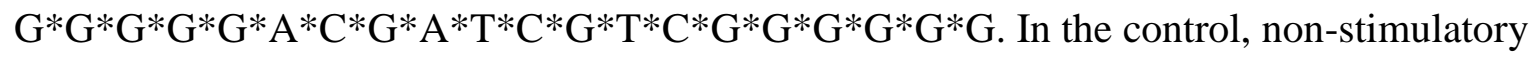
ODN (GpC) the places of the cytosines and guanines in the CpG-B sequence are switched. LPS from E. coli 0111:B4 was obtained from Sigma Aldrich (cat.\# L2630). The p38 inhibitor (SB203580) was purchased from Alexis Biochemicals. Wortmannin was purchased from Invivogen. Rabbit antibodies against phosphorylated p38 (p-p38), p-MKK3/6, p-MK2, pERK, p-Akt and eukaryotic elongation factor 2 (eEF2) were obtained from Cell Signaling Technology. The secondary horseradish peroxidase (HRP)-conjugated goat anti-rabbit IgGHRP was purchased from Santa Cruz Biotechnology

\subsection{Isolation and stimulation of primary mononuclear phagocytes from Atlantic salmon}

Head kidney (HK) leukocytes were isolated as described previously [10]. The organs were passed through a 100-mm pore size cell strainer (Falcon) in L-15 medium containing penicillin $(60 \mathrm{mg} / \mathrm{ml})$, streptomycin $(100 \mathrm{mg} / \mathrm{ml}), 2 \%$ foetal bovine serum (FBS) and heparin (20U/ml). The resulting suspension was placed on a 25/54\% discontinuous Percoll gradient and centrifuged at $400 \mathrm{~g}$ for $40 \mathrm{~min}$ at $4^{\circ} \mathrm{C}$. The cells at the interface were collected and washed twice in L-15 medium. The cells were seeded in 24 -well plates at a density of $7 \times 10^{6}$ 
cells per well. After 1 hour of incubation in L-15, supplemented with $0.1 \%$ FBS at $14^{\circ} \mathrm{C}$, the cells were washed by vigorous pipetting with fresh L15 medium and the adherent mononuclear phagocytes were further incubated in L-15 supplemented with 5\% FBS. In the different experiments the cells were stimulated immediately after washing or after 1, 3 and 5 days as indicated in the Results section and in the figure legends. For the Western blot analysis, the cells were lysed with $50 \mu \mathrm{l}$ of NuPAGE LDS sample buffer, sonicated and heated for $10 \mathrm{~min}$ at $70^{\circ} \mathrm{C}$. For RT-PCR microarray analyses, the cells were lysed with TRIzol Reagent (Invitrogen).

\subsection{Western blot analysis}

Cell lysates were separated by SDS/PAGE (4-12\% precast NuPAGE; Invitrogen), followed by transfer to a $0.45 \mu \mathrm{m}$ pore size polyvinylidene difluoride membrane (Millipore). The blots were pre-blocked for 1 hour (Tris-buffered saline, 5\% BSA, 0.1\% Tween-20) and incubated for 24 hours with 1:1000 dilution of the primary antibodies followed by 1 hour incubation with 1:10000 dilution of the secondary antibody in blocking solution. Detection was performed with Super-Signal West Pico substrate (Pierce Biotechnology). The membranes were stripped for $10 \mathrm{~min}$ in $0.2 \mathrm{M} \mathrm{NaOH}$ followed by washing, blocking and antibody incubation. The size of the proteins was estimated using the MagicMark Western protein standard (Invitrogen).

\subsection{Real-time PCR}

The RNA isolation and the cDNA synthesis were performed with RNeasy Mini Kit (Qiagen) and TaqMan Reverse Transcription Reagents kit (Applied Biosystems) as previously described ([11]). The Real-time PCR reactions were assembled using Power SYBR Green PCR Master Mix (Applied Biosystems). The primer sequences, the reaction conditions and 
the data analysis have previously been described [12]. Statistical analysis was carried out using the Student t test.

2.5. Microarray analysis

The cDNA microarray containing 1800 unique clones, the reagents and the procedures have been previously described [12]. 


\section{Results}

\subsection{Time course of the MK2, p38, ERK and MKK3/6 phosphorylation in CpG and LPS-}

stimulated mononuclear phagocytes; the p38 inhibitor effectively inhibits the CpG-and LPSinduced MK2 phosphorylation

In order to analyze the efficacy of the p38 inhibitor, the cells were stimulated alone or in the presence of the inhibitor for $0.5,2$ and 4 hours prior to assessment of the phosphorylation of MK2 which is a target of p38 (Fig. 1). The CpG-B and the LPS-induced MK2 phosphorylation were completely suppressed in the presence of the p38 inhibitor at all of the tested time points. These data also showed that the LPS stimulation induced a faster response and there was a good correlation between the levels of phospho-p38 (p-p38) and pMK2 in cells stimulated in the absence of the p38 inhibitor.

It has been previously shown that salmon MKK6 is involved in the activation of p38 [13] .In the current study, the LPS stimulation induced the phosphorylation of MKK6b/c and the putative MKK4 at all time points. The CpG stimulation upregulated detectably pMKK6b/c only at the latest time point (4 hours). The band corresponding to salmon pMKK6a, which migrates slightly faster than p-MKK6b/c [13], was observed in all of the samples and it was not detectably influenced by any of the treatments. Interestingly, the p38 inhibitor induced the MKK6b/c phosphorylation at all of the time points suggesting that $\mathrm{p} 38$ may exert a negative feedback on the MKK6b/c activity.

Unlike the p38 whose activity is mostly controlled by stress and proinflammatory stimuli [5], the activation of the ERK is controlled primarily by mitogens which signal through receptors involved in cell growth, proliferation and differentiation including Gprotein coupled receptors, receptor tyrosine kinases, Integrins, and Ion Channels [14, 15]. Surprisingly, the CpG stimulation down-regulated the levels of p-ERK at all sampling time points indicating that this process is not transient but it is both fast and relatively long-lasting 
(Fig. 1). Like MKK6b/c, the p38 inhibitor upregulated p-ERK in CpG-treated samples at 30 min and 2 hours of stimulation, while at the latest time-point (4 hours) positive effect was not clearly visible.

\subsection{Microarray analysis - influence of the p38 inhibitor SB203580 on the CpG and LPS-}

modulated gene expression in salmon mononuclear phagocytes.

The microarray data shown in Fig. 1A are derived from a larger experiment and it should be pointed out that the values for the gene expression in cells stimulated with $\mathrm{CpG}$ and LPS in the absence of the p38 inhibitor have been previously published [12]. In the current study they have been used solely as control values for the effect of SB203580.

The chosen experimental conditions, including the concentrations of the stimulants (2 $\mu \mathrm{M}$ CpG ODNs and $50 \mu \mathrm{g} / \mathrm{ml}$ of LPS $)$ and the p38 inhibitor $(10 \mu \mathrm{M})$ and the preincubation period with the inhibitor (1 hour) were based on a previous study [6] .

The microarray results in the current study demonstrate that the upregulation of most of the CpG and LPS-inducible transcripts was affected by SB203580 which highlighted the importance of the p38 signaling for the activation of the cells by these immunostimulants. Most of the genes that were weakly upregulated by the PAMP stimulation were completely suppressed by the inhibitor. Of note, DUSP5 which is involved in MAPK dephosphorylation [16] was highly upregulated by the PAMPs and it was completely suppressed in the presence of the p38 inhibitor indicating its induction by CpGs and LPS might be a part of a negative feedback mechanism. On the other hand, other highly-inducible genes involved in regulation of NF-kappaB activation, including two NF-kappaB inhibitor alpha isoforms, and also p62 and TRAF2 were partially inhibited suggesting involvement of p38-independent signaling pathways in their induction by PAMPs 
The microarray analyses were performed with pooled RNA from individually stimulated samples from 3 fish. SYBR Green Real time PCR (RT-QPCR) was further used to validate microarray results using the individual RNA samples. The results shown in Fig. 2B confirmed that the p38 inhibitor consistently suppressed the CpG- and LPS-induced upregulation of the surface marker gene CD40, the macrophage inflammatory protein CCL4 and MMP9. In addition, IL-1 $\beta$, an important proinflammatory cytokine was also suppressed by the $\mathrm{p} 38$ inhibitor.

\subsection{CpG-induced p-ERK downregulation and p-p38 upregulation-dose response}

Salmon mononuclear phagocytes were stimulated with increasing CpG ODN concentrations for 2 hours prior to assessment of the p-ERK and p-p38 levels. The western blot results shown in Fig. 3 indicate that the p-ERK level is slightly downregulated even in cells stimulated with the lowest concentration of CpG ODNs $(0.5 \mu \mathrm{M})$ and the intensity of the p-ERK band further declines with increased CpG ODN concentrations. The blot was also reprobed with the p-p38 antibody to confirm the cell activation. The p-p38 band was induced by $\mathrm{CpG}$ ODNs at $2 \mu \mathrm{M}$ and higher concentrations.

\subsection{The intensity of ERK phosphorylation in cultured mononuclear phagocytes correlates} with PI3K activity and the spontaneous morphological differentiation of mononuclear phagocytes.

In the current study, preliminary experiments indicated that ERK and p38 phosphorylation might be influenced significantly by the length of in vitro incubation preceding the activation of the cells with PAMPs. The results presented in Fig.4A demonstrate that the p-ERK levels in non-stimulated cells increased gradually between day 1 and day 5 of in vitro incubation and correlated with the phosphatidylinositol 3-kinase (PI3K) 
activity as indicated by the increased levels of p-Akt, which is a downstream target of PI3K. Induction of ERK phosphorylation by $\mathrm{CpGs}$ and inverted (GpC), control ODNs could inconsistently be detected on day 1 when p-ERK levels were relatively low. In contrast, after 3-5 days of incubation, stimulation with both ODN types exerted a negative effect on the pERK levels. The ERK phosphorylation in resting cells was highly sensitive to wortmannin indicating it was PI3K-dependent. In contrast, the p38 phosphorylation was not detectably affected by the PI3K inhibitor. The CpG-induced p38 phosphorylation was affected by the length of in vitro incubation and it was substantially lower on day 3 and barely detectable on day 5 .

It has been previously demonstrated that trout mononuclear phagocytes differentiate spontaneously in vitro and over the course of several days develop a mature, macrophage-like phenotype $[12,17]$. In the current study the morphology of the cells was examined under a microscope at each time point prior to taking the samples for Western blot (WB) (Fig. 3B). Similar to trout, salmon mononuclear phagocytes were also able to differentiate spontaneously as shown by development of a macrophage-like morphology within 5 days of in vitro culture. 


\section{Discussion}

The pyridinyl imidazole SB203580 has been described as a specific p38 inhibitor and has been widely used in studies aimed to characterize the function of this kinase [8].The data presented in the current study show that it effectively inhibits the PAMP-induced p38 activity in primary salmon mononuclear phagocytes and highlight the role of p38 in the response of these cells to CpG ODNs and LPS. SB203580 acts on p38 by competitive binding within the ATP pocket but does not inhibit phosphorylation of p38 MAPK by upstream kinases [18]. Therefore, the efficacy of the inhibitor was confirmed by its potential to completely suppress the CpG- and LPS-induced phosphorylation of MK2, a p38 substrate [8]. The data correlates well with an earlier study, showing that the same inhibitor totally prevented phosphorylation of MK2 in sodium arsenite stressed CHSE-cells, in which p-p38 was upregulated [13]

In mammals MKK3, MKK6 and, in some cases, MKK4 are activators of p38 [19], however, in fish the activation of p38 by upstream kinases is poorly understood. A study has shown that teleosts lack MKK3 and salmon MKK6 homologs are involved in stress-induced phosphorylation of p38 [13]. Nevertheless, the results in this study also suggested that salmon p38 may be activated by additional kinases depending on the type of the stimulus. In the current study, the intensity of the p-MKK6 and the putative p-MKK4 bands did not unambiguously correlate with the level of p38 phosphorylation. MKK6a was constitutively phosphorylated whereas MKK6b/c (very likely MKK6c, since a previous study has shown that salmon macrophages do not express MKK6b mRNA [13]) and the putative MKK4 were weakly and inconsistently phosphorylated following LPS and CpG stimulation. These results indicate that other kinase/s may be involved in the activation of p38 by PAMPs in salmon mononuclear phagocytes.

In contrast with its inhibitory effect on p38 activity, SB203580 upregulated pMKK6b/c and had a positive effect on p-ERK levels in CpG-treated cells. It has for a long time been 
known that p38 activates a negative feedback loop which in addition to p38 also affects ERK and JNK activity [20]. Upregulation of various phosphatases such as dual specificity phosphatases (DUSP) is an important mechanism through which MAPK activity is controlled [21], [22]. In the current study the CpG- and LPS-induced upregulation of DUSP5, a molecule which in mammals specifically dephosphorylates ERK, appeared to be completely p38dependent as indicated by the microarray analysis and may have been involved in negative regulation of ERK activity by p38. On the other hand, it should also be considered that SB203580 has potential to stimulate Raf1 [23]. This kinase is involved in ERK activation and a model has shown that it may also be involved in MKK6 phosphorylation [24]. Therefore the relatively fast SB203580-induced phosphorylation of salmon MKK6b/c and ERK may also be due to a direct positive effect of the inhibitor on Raf-1 or other upstream signalling components.

Upregulation of ERK phosphorylation by SB203580 was detected only in CpGstimulated cells in which the constitutive p-ERK levels were, surprisingly, downregulated. This downregulation was not dependent on PAMP-induced p38 activity since LPS upregulated faster p38 phosphorylation as compared to CpGs but it did not suppress the pERK levels at any of the assayed time points. In studies with mammalian antigen-presenting cells it has been shown that PAMPs, such as CpGs and LPS induce phosphorylation of both p38 and ERK [25] [26]. However, another study has demonstrated that, following in vitro differentiation for several days, stimulation of murine dendritic cells with CpG ODNs, but not LPS, suppressed ERK activity [27]. It has been shown that in vitro cultured trout HK-derived adherent monocytes differentiate spontaneously and following incubation for several days develop a more mature, macrophage-like phenotype. This is manifested by changes in their morphology and enhanced potential to respond to LPS stimulation [17, 28, 29]. In the current study, the non-stimulated salmon primary cells displayed similar morphological changes 
when cultured in vitro for a period of 3 to 5 days (Fig. 3 B). It is very likely that the observed in vitro differentiation of salmonid mononuclear phagocytes depends on the action of autocrine growth factors, such as the macrophage colony stimulating factor (M-CSF) which is necessary for the development and the differentiation of mononuclear phagocytes to mature macrophages [30]. The action of M-CSF is mediated by ERK [31] and PI3K/Akt-mediated signaling cascades [32]. The gradually increasing levels of p-ERK and p-Akt (a downstream mediator of PI3K signalling) over the course of in vitro incubation correlated with the morphological differentiation of the salmon mononuclear phagocytes and could be explained by the activity of such growth factors. It has also been shown that, in mouse osteoclasts the M-CSF-induced phosphorylation of ERK is dependent on upstream PI3K activation [33]. Therefore, it is likely that the high sensitivity of ERK phosphorylation to wortmannin, a specific PI3K inhibitor [34], and the relatively good correlation between the levels of phosphorylated ERK and Akt might reflect a similar interplay between these two kinases in salmon mononuclear phagocytes as well.

In the current study, suppression of ERK phosphorylation by $\mathrm{CpG}$ and non-stimulatory GpC ODNs was observed only in cells incubated in vitro for at least 3 days. The p-ERK levels in ODN-treated cells were not down-regulated below these detected in control cells on day 1 indicating the negative effect might be due to suppression of growth factor receptor/s signalling. Interestingly, stimulation with bacterial DNA was shown to quickly downregulate the M-CSF receptor in murine macrophages [35]. However, the same effect was not induced by non-stimulatory calf thymus DNA and therefore, the observation that the p-ERK downregulation in salmon mononuclear phagocytes was also observed following treatment of with control ODNs would be difficult to explain with this phenomenon and it, most likely, depends on a different mechanism/s. 
Another unexpected observation was that the $\mathrm{CpG}$-induced p38 phosphorylation appeared to be gradually supressed throughout the course of in vitro incubation. It is possible that this might be due to changes in the kinetics of p38 activation - either a transient peak or a delayed phosphorylation. However, another explanation is the potential autocrine action of M$\mathrm{CSF}$, or analogous growth factors, since in primary murine macrophages the p38 activation by CpGs, but not LPS, was suppressed by pretreatment with M-CSF which at the same time upregulated ERK phosphorylation [36]. In addition, it has been shown that the PI3K signalling suppresses TLR-induced p38 phosphorylation in innate immune cells including DCs [37] and macrophages [38]. Enhanced activity of PI3K in macrophages results in increased DUSP1 (a p38 specific phosphatase) expression, IL-10 production and, as a result, diminished p38 activation and lowered production of proinflammatory cytokines in response to pathogens [39].

In summary, the data presented in the current study indicate that $\mathrm{p} 38$ signalling is crucial for activation of salmon mononuclear phagocytes by CpG ODNs and LPS. In contrast to p38, ERK is constitutively phosphorylated in vitro through a mechanism that is, most likely, PI3Kdependent. Explaining the downregulation of p-ERK by both $\mathrm{CpG}$ and control ODNs may be an interesting direction for future research. 


\section{Acknowledgements}

We thank H.L. Thim for technical assistance. This study was supported by Aquaculture program of The Research Council of Norway (Grant no 172253/S40 and 183196/S40 InNoVacc). 


\section{References}

1. West MA, Wallin RP, Matthews SP, Svensson HG, Zaru R, Ljunggren HG, et al. Enhanced dendritic cell antigen capture via toll-like receptor-induced actin remodeling. Science. 2004 305:1153-7.

2. Zaru R, Ronkina N, Gaestel M, Arthur JS, Watts C. The MAPK-activated kinase Rsk controls an acute Toll-like receptor signaling response in dendritic cells and is activated through two distinct pathways. Nat Immunol. 2007 8:1227-35.

3. Chen Z, Gibson TB, Robinson F, Silvestro L, Pearson G, Xu B, et al. MAP kinases. Chem Rev. 2001 101:2449-76.

4. Rao KM. MAP kinase activation in macrophages. J Leukoc Biol. 2001 69:3-10.

5. Raingeaud J, Whitmarsh AJ, Barrett T, Derijard B, Davis RJ. MKK3- and MKK6regulated gene expression is mediated by the $\mathrm{p} 38$ mitogen-activated protein kinase signal transduction pathway. Mol Cell Biol. 1996 16:1247-55.

6. Hansen TE, Jørgensen JB. Cloning and characterisation of p38 MAP kinase from Atlantic salmon: A kinase important for regulating salmon TNF-2 and IL-1[beta] expression. Mol Immunol. 2007 44:3137-46.

7. Holen E, Winterthun S, Du ZY, Krovel AV. Inhibition of p38 MAPK during cellular activation modulate gene expression of head kidney leukocytes isolated from Atlantic salmon (Salmo salar) fed soy bean oil or fish oil based diets. Fish Shellfish Immunol. 2011 30:397405.

8. Cuenda A, Rouse J, Doza YN, Meier R, Cohen P, Gallagher TF, et al. SB 203580 is a specific inhibitor of a MAP kinase homologue which is stimulated by cellular stresses and interleukin-1. FEBS Lett. 1995 364:229-33.

9. Yong HY, Koh MS, Moon A. The p38 MAPK inhibitors for the treatment of inflammatory diseases and cancer. Expert Opin Investig Drugs. 2009 18:1893-905.

10. Strandskog G, Ellingsen T, Jørgensen JB. Characterization of three distinct CpG oligonucleotide classes which differ in ability to induce IFN alpha/beta activity and cell proliferation in Atlantic salmon (Salmo salar L.) leukocytes. Developmental and comparative immunology. 2007 31:39-51.

11. Strandskog G, Villoing S, Iliev DB, Thim HL, Christie KE, Jørgensen JB.

Formulations combining $\mathrm{CpG}$ containing oliogonucleotides and poly I:C enhance the magnitude of immune responses and protection against pancreas disease in Atlantic salmon. Developmental and comparative immunology. 2011 35:1116-27.

12. Iliev DB, Jorgensen SM, Rode M, Krasnov A, Harneshaug I, Jørgensen JB. CpGinduced secretion of MHCIIbeta and exosomes from salmon (Salmo salar) APCs. Dev Comp Immunol. 2010 34:29-41.

13. Hansen TE, Puntervoll P, Seternes OM, Jorgensen JB. Atlantic salmon possess three mitogen activated protein kinase kinase 6 paralogs responding differently to stress. Febs Journal. 2008 275:4887-902.

14. Roberts PJ, Der CJ. Targeting the Raf-MEK-ERK mitogen-activated protein kinase cascade for the treatment of cancer. Oncogene. 2007 26:3291-310.

15. Pearson G, Robinson F, Beers Gibson T, Xu BE, Karandikar M, Berman K, et al. Mitogen-activated protein (MAP) kinase pathways: regulation and physiological functions. Endocr Rev. 2001 22:153-83. 
16. Kovanen PE, Rosenwald A, Fu J, Hurt EM, Lam LT, Giltnane JM, et al. Analysis of gamma c-family cytokine target genes. Identification of dual-specificity phosphatase 5

(DUSP5) as a regulator of mitogen-activated protein kinase activity in interleukin-2 signaling.

J Biol Chem. 2003 278:5205-13.

17. MacKenzie S, Planas JV, Goetz FW. LPS-stimulated expression of a tumor necrosis factor-alpha mRNA in primary trout monocytes and in vitro differentiated macrophages. Developmental and comparative immunology. 2003 27:393-400.

18. Kumar S, Jiang MS, Adams JL, Lee JC. Pyridinylimidazole compound SB 203580 inhibits the activity but not the activation of p38 mitogen-activated protein kinase. Biochem Biophys Res Commun. 1999 263:825-31.

19. Brancho D, Tanaka N, Jaeschke A, Ventura JJ, Kelkar N, Tanaka Y, et al. Mechanism of p38 MAP kinase activation in vivo. Genes Dev. 2003 17:1969-78.

20. Wang X, Rao J, Studzinski GP. Inhibition of p38 MAP kinase activity up-regulates multiple MAP kinase pathways and potentiates 1,25-dihydroxyvitamin $\mathrm{D}(3)$-induced differentiation of human leukemia HL60 cells. Exp Cell Res. 2000 258:425-37.

21. Lang R, Hammer M, Mages J. DUSP meet immunology: dual specificity MAPK phosphatases in control of the inflammatory response. J Immunol. 2006 177:7497-504.

22. Patterson KI, Brummer T, O'Brien PM, Daly RJ. Dual-specificity phosphatases: critical regulators with diverse cellular targets. Biochem J. 2009 418:475-89.

23. Kalmes A, Deou J, Clowes AW, Daum G. Raf-1 is activated by the p38 mitogenactivated protein kinase inhibitor, SB203580. FEBS Lett. 1999 444:71-4.

24. Wang W, Chen JX, Liao R, Deng Q, Zhou JJ, Huang S, et al. Sequential activation of the MEK-extracellular signal-regulated kinase and MKK3/6-p38 mitogen-activated protein kinase pathways mediates oncogenic ras-induced premature senescence. Mol Cell Biol. 2002 22:3389-403.

25. Yi AK, Yoon JG, Yeo SJ, Hong SC, English BK, Krieg AM. Role of mitogenactivated protein kinases in CpG DNA-mediated IL-10 and IL-12 production: central role of extracellular signal-regulated kinase in the negative feedback loop of the CpG DNA-mediated Th1 response. J Immunol. 2002 168:4711-20.

26. Kawai T, Adachi O, Ogawa T, Takeda K, Akira S. Unresponsiveness of MyD88deficient mice to endotoxin. Immunity. 1999 11:115-22.

27. Hacker H, Mischak H, Hacker G, Eser S, Prenzel N, Ullrich A, et al. Cell type-specific activation of mitogen-activated protein kinases by CpG-DNA controls interleukin-12 release from antigen-presenting cells. Embo J. 1999 18:6973-82.

28. Iliev DB, Roach JC, Mackenzie S, Planas JV, Goetz FW. Endotoxin recognition: in fish or not in fish? FEBS Lett. 2005 579:6519-28.

29. Iliev DB, Liarte CQ, MacKenzie S, Goetz FW. Activation of rainbow trout (Oncorhynchus mykiss) mononuclear phagocytes by different pathogen associated molecular pattern (PAMP) bearing agents. Mol Immunol. 2005 42:1215-23.

30. Chitu V, Stanley ER. Colony-stimulating factor-1 in immunity and inflammation. Curr Opin Immunol. 2006 18:39-48.

31. Gobert Gosse S, Bourgin C, Liu WQ, Garbay C, Mouchiroud G. M-CSF stimulated differentiation requires persistent MEK activity and MAPK phosphorylation independent of Grb2-Sos association and phosphatidylinositol 3-kinase activity. Cell Signal. 2005 17:135262.

32. Varticovski L, Druker B, Morrison D, Cantley L, Roberts T. The colony stimulating factor-1 receptor associates with and activates phosphatidylinositol-3 kinase. Nature. 1989 342:699-702. 
33. Bradley EW, Ruan MM, Vrable A, Oursler MJ. Pathway crosstalk between Ras/Raf and PI3K in promotion of M-CSF-induced MEK/ERK-mediated osteoclast survival. J Cell Biochem. 2008 104:1439-51.

34. Arcaro A, Wymann MP. Wortmannin is a potent phosphatidylinositol 3-kinase inhibitor: the role of phosphatidylinositol 3,4,5-trisphosphate in neutrophil responses.

Biochem J. 1993296 ( Pt 2):297-301.

35. Sester DP, Beasley SJ, Sweet MJ, Fowles LF, Cronau SL, Stacey KJ, et al.

Bacterial/CpG DNA down-modulates colony stimulating factor-1 receptor surface expression on murine bone marrow-derived macrophages with concomitant growth arrest and factorindependent survival. J Immunol. 1999 163:6541-50.

36. Sweet MJ, Campbell CC, Sester DP, Xu D, McDonald RC, Stacey KJ, et al. Colonystimulating factor-1 suppresses responses to CpG DNA and expression of toll-like receptor 9 but enhances responses to lipopolysaccharide in murine macrophages. J Immunol. 2002 168:392-9.

37. Fukao T, Tanabe M, Terauchi Y, Ota T, Matsuda S, Asano T, et al. PI3K-mediated negative feedback regulation of IL-12 production in DCs. Nat Immunol. 2002 3:875-81.

38. Medina EA, Morris IR, Berton MT. Phosphatidylinositol 3-kinase activation attenuates the TLR2-mediated macrophage proinflammatory cytokine response to Francisella tularensis live vaccine strain. J Immunol. 2010 185:7562-72.

39. Gunzl P, Bauer K, Hainzl E, Matt U, Dillinger B, Mahr B, et al. Anti-inflammatory properties of the PI3K pathway are mediated by IL-10/DUSP regulation. J Leukoc Biol. 2010 88:1259-69. 


\section{Figure captions}

Figure 1. Time course of the MK2, p38, ERK and MKK3/6 phosphorylation in CpG and LPS-stimulated mononuclear phagocytes; the p38 inhibitor effectively inhibits the CpG- and LPS-induced MK2 phosphorylation at all of the tested time points. The cells were incubated in vitro for 3 days prior to stimulation with $\mathrm{CpG}$ and LPS alone or in presence of the p38 inhibitor prior to sample harvesting and WB analysis with the indicated Abs. Reprobing with the eEF2 Ab was used as a loading control Representative data from experiments with cells isolated from two individuals are shown

Figure 2. The p38 ihibitor SB203580 interferes with the upregulation of most of the CpG- and LPS-induced transcripts in salmon mononuclear phagocytes. A, Microarray analysis of cells stimulated with CpG ODNs and LPS for 4 hours. The values show the fold upregulation as compared to non-stimulated controls. The color intensity correlates with the level of upregulation. The microarray was probed with pooled RNA from individually stimulated samples from 3 individuals. $B$, The negative effect of $\mathrm{p} 38$ inhibition of immune genes was confirmed with SYBR Green Real Time PCR. The samples from the 3 individuals used for the microarray were analyzed separately. The values are mean fold upregulation as compared to non-stimulated cells. Statistical analysis was carried out using the Student t test $(* \mathrm{P}<0.05)$.

Figure 3. CpG ODN-induced p-ERK downregulation and p-p38 upregulation dose response. The cells were incubated in vitro for 3 days prior to stimulation with different concentrations of $\mathrm{CpG}$ ODNs for 2 hours and WB analysis with p-ERK and p-p38 antibodies. Reprobing with the eEF2 Ab was used as a loading control. Representative data from experiments with cells isolated from two individuals are shown 
Figure 4. The intensity of ERK phosphorylation in in vitro cultured mononuclear phagocytes correlates with PI3K activity and the spontaneous morphological differentiation of mononuclear phagocytes. The cells on day 1 were stimulated immediately after removal of non-adherent cells (1 hour after seeding the whole HK population). The stimulations were performed with $2 \mu \mathrm{M}$ concentration of $\mathrm{CpG}$ ODNs and inverted, control GpC ODNs for 2 hours prior to isolation of proteins and WB analysis with the indicated Abs (panel A) or microscopy imaging (panel B). In order to block PI3K activity, on day 5, the cells were pretreated for 1 hour with $2 \mu \mathrm{M}$ Wortmannin prior to stimulation as indicated. Representative data from experiments with cells isolated from two individuals are shown. 
Figure 1

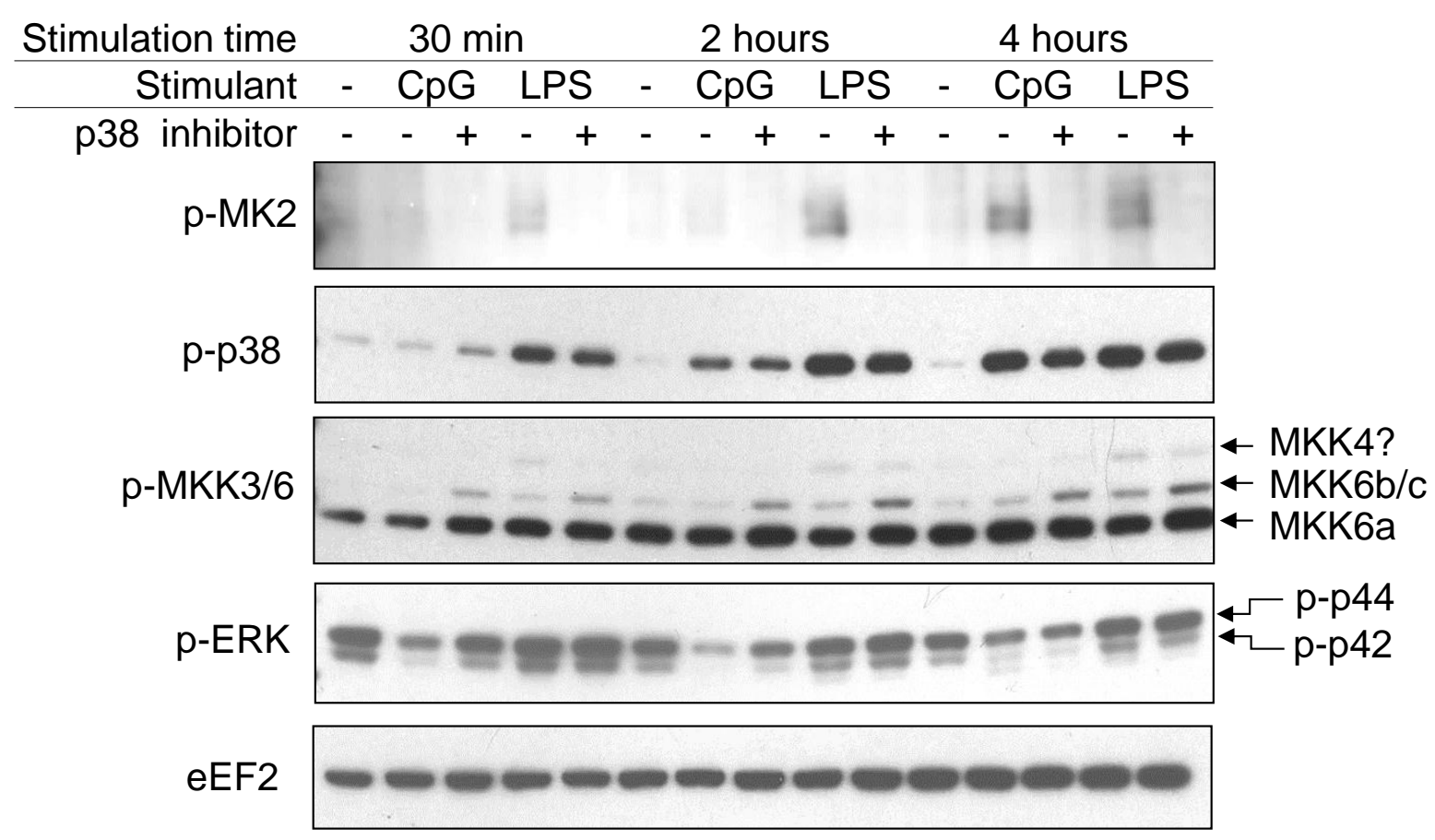


Figure 2

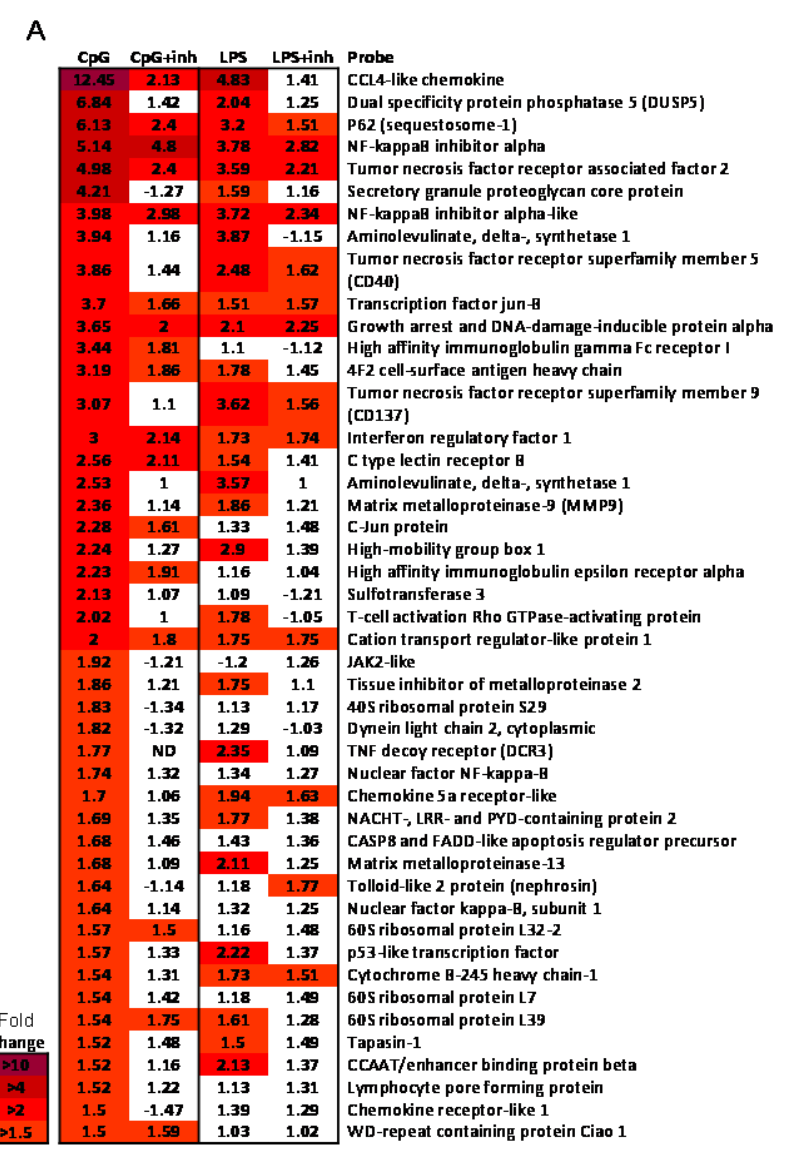

B
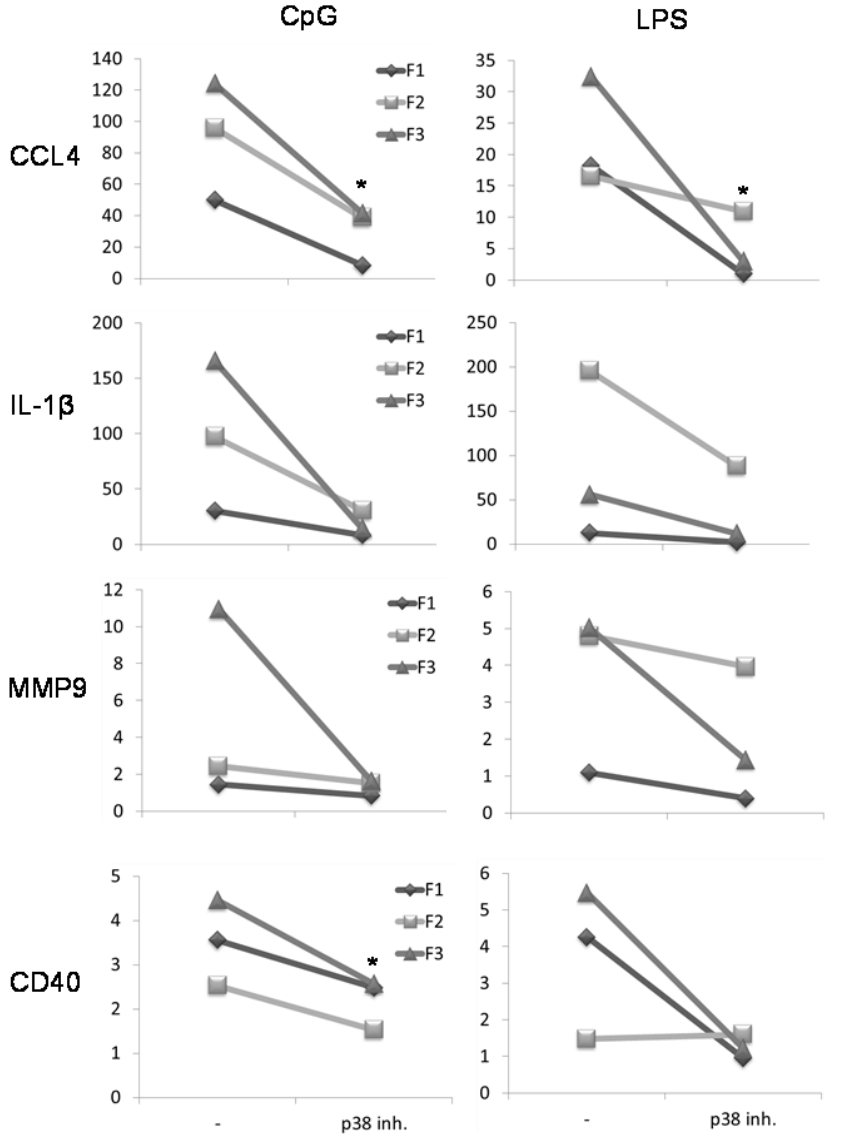
Figure 3

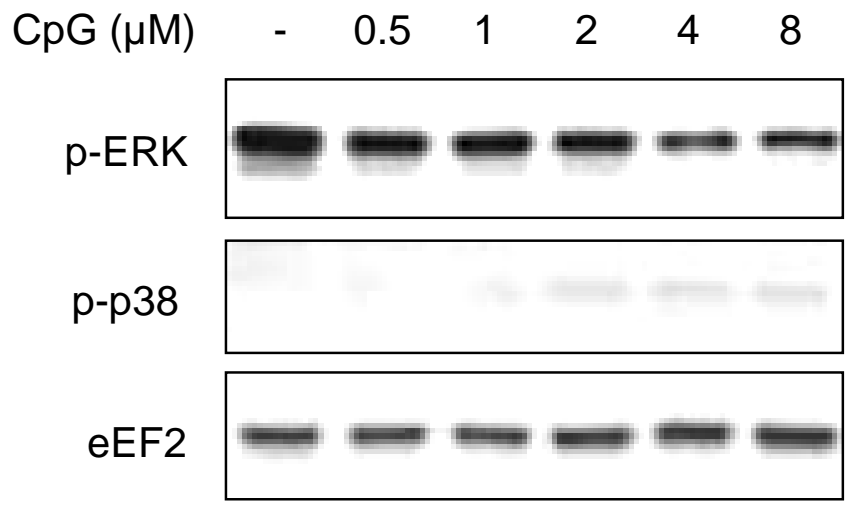


Figure 4

A

\begin{tabular}{|c|c|c|c|c|c|c|c|c|c|c|c|}
\hline & & day 1 & & & day 3 & & & & day 5 & & \\
\hline Stimulation & - & $\mathrm{GpC}$ & CpG & - & $\mathrm{GpC}$ & CpG & - & - & GpC & $\mathrm{CpG}$ & $\mathrm{CpG}$ \\
\hline Wotmannin & - & - & - & - & - & - & - & + & - & - & + \\
\hline phospho-ERK & + & $\stackrel{0}{\longrightarrow}$ & $=4$ & 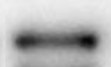 & $m$ & 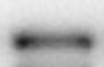 & $\theta$ & & . & 5 & \\
\hline Phospho-Akt & 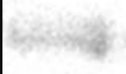 & 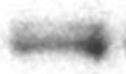 & weve & twis & $\frac{1}{2}$ & 40 & & & & . & \\
\hline
\end{tabular}
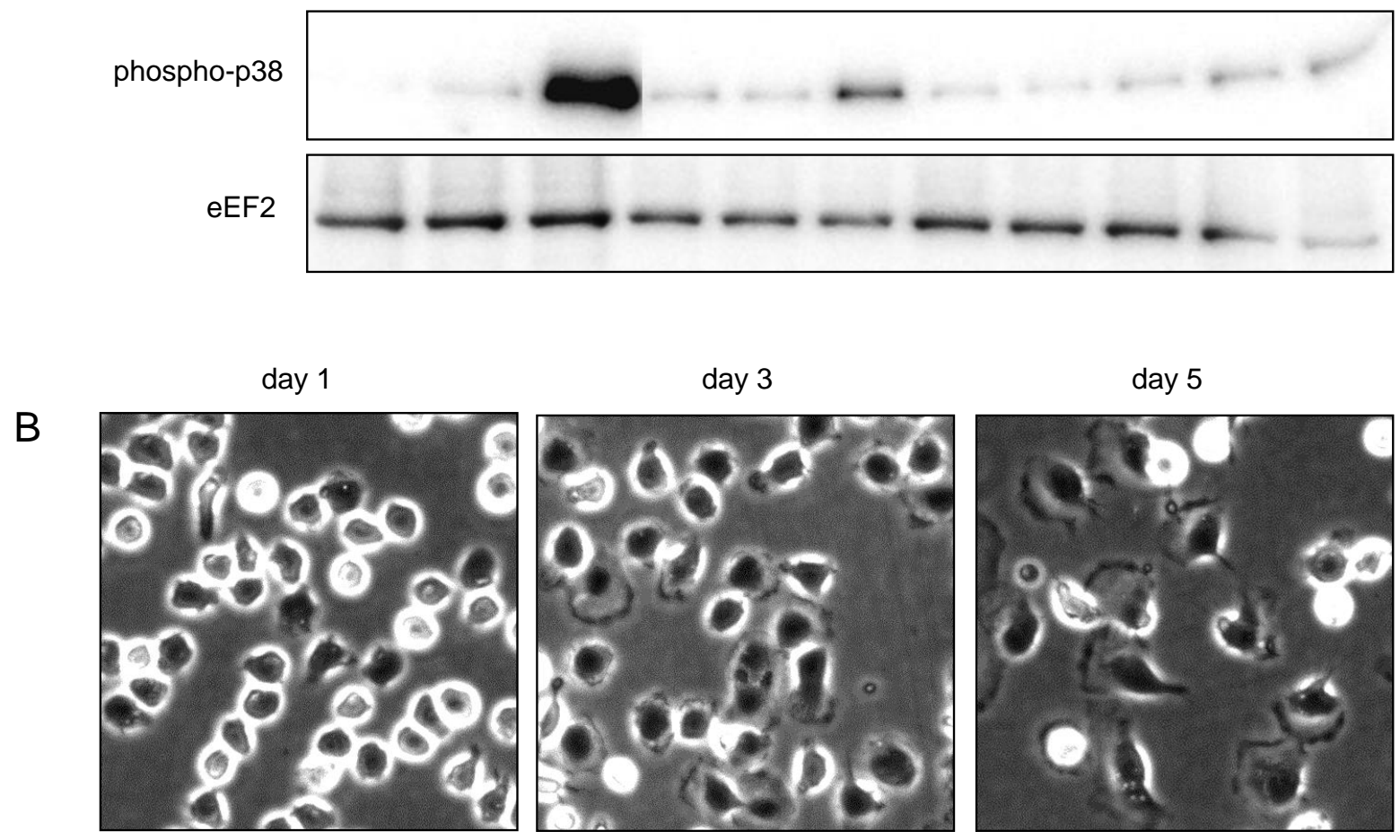